\title{
Charge renormalization and inversion of a highly charged lipid bilayer: Effects of dielectric discontinuities and charge correlations
}

\author{
Sattar Taheri-Araghi and Bae-Yeun Ha \\ Department of Physics, University of Waterloo, Ontario N2L 3G1, Canada
}

(Received 18 February 2005; published 19 August 2005)

\begin{abstract}
We reexamine the problem of charge renormalization and inversion of a highly charged surface of a low dielectric constant immersed in ionic solutions. To be specific, we consider an asymmetrically charged lipid bilayer, in which only one layer is negatively charged. In particular, we study how dielectric discontinuities and charge correlations (among lipid charges and condensed counterions) influence the effective charge of the surface. When counterions are monovalent (e.g., $\mathrm{Na}^{+}$), our mean-field approach implies that dielectric discontinuities can enhance counterion condensation. A simple scaling picture shows how the effects of dielectric discontinuities and surface-charge distributions are intertwined: Dielectric discontinuities diminish condensation if the backbone charge is uniformly smeared out while counterions are localized in space; they can, however, enhance condensation when the backbone charge is discrete. In the presence of asymmetric salts such as $\mathrm{CaCl}_{2}$, we find that the correlation effect, treated at the Gaussian level, is more pronounced when the surface has a lower dielectric constant, inverting the sign of the charge at a smaller value of $\mathrm{Ca}^{2+}$ concentration.
\end{abstract}

DOI: 10.1103/PhysRevE.72.021508

PACS number(s): 61.20.Qg, 82.70.-y, 87.15.-v

\section{INTRODUCTION}

Macromolecules such as DNA and biomembranes carry a large number of charges in aqueous solution interacting with other ions. They can thus trap oppositely charged ions (counterions) in their close proximity under a variety of conditions; some of them are irreversibly adsorbed onto the surface, forming the so-called stern layer, but others are less tightly bound to the surface, forming a diffusive layer of excess counterions $[1,2]$. While permanently adsorbed ions in the Stern layer can be considered as part of surface charges, the diffusive layer is a dynamic structure, constantly exchanging ions with those in bulk. Nevertheless, it has proven to be useful to consider the macroion and its diffusive layer as forming a single object, which is often referred to as a "dressed ion" $[3,4]$. It has long been recognized that counterions in the diffusive layer play an important role in regulating the charge properties of macroions as in the electrostatic binding and transport of macroions [5-7]. It is thus of practical importance to study how their physical properties can be controlled by experimentally accessible parameters such as ionic strength, ion valences, and dielectric properties.

In this paper, we study the (reversible) electric binding of counterions onto an oppositely charged surface in an aqueous solution. To be specific, we consider a negatively charged lipid bilayer of thickness $d$ and a dielectric constant $\epsilon_{<}$, immersed in electrolyte solutions of a dielectric constant $\epsilon_{>}$, as illustrated in Fig. 1. (The subscripts $>$ and $<$ are a reminder that $\epsilon_{>}$is typically larger than $\epsilon_{<}$.) The bilayer is assumed to be asymmetrically charged: one of the layers is negatively charged with a charge density $-e \sigma_{0}$ and the other one is neutral (as in red blood cell membranes [8]). In addition to monovalent salts (e.g., $\mathrm{NaCl}$ ), there can be $Z: 1$ salts (e.g., $\mathrm{CaCl}_{2}$ ). The charged surface (at $x=0$ ) can attract counterions (e.g., $\mathrm{Na}^{+}$or $\mathrm{Ca}^{2+}$ ) and trap them in its close proximity. This phenomenon, often referred to as counterion condensation, results in a renormalization of the surface charge $[1,2]$. Un- der certain conditions, the sign of the renormalized charge can be inverted; this phenomenon is known as "charge inversion" [9-11].

In particular, we study how dielectric discontinuities influence the electric binding of counterions or simply counterion condensation (thus charge inversion). Our main focus will be laid on the computation of renormalized charges rather than on the detailed structure of the diffusive layer. We first tackle this problem at the mean-field level. To this end, we use two seemingly distinct methods: a two-state model $[1,2]$ and a matching method [12]. In the latter case, the Poisson-Boltzmann (PB) equation is matched, at large distances, with the corresponding Debye-Hückel (DH) equation (or the linearized PB equation) with a renormalized charge

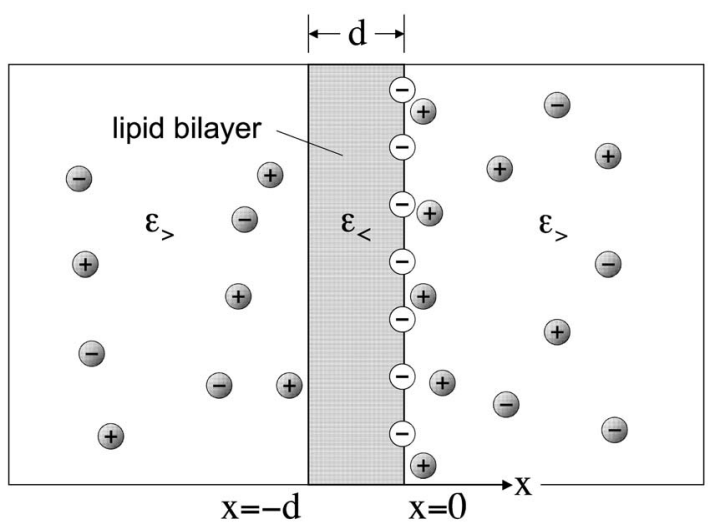

FIG. 1. Schematic view of a negatively charged lipid bilayer of thickness $d$ immersed in an ionic solution. The dielectric constant of the bilayer $\epsilon_{<}$is typically smaller than that of the solution $\epsilon_{>}$. The bilayer is assumed to be asymmetrically charged: the left side of the plate at $x=-d$ is neutral while the other side at $x=0$ is negatively charged with a charge density $-e \sigma_{0}$. The resulting system resembles an asymmetrically charged cell membrane (e.g., red blood cell membranes). 
[12]. Both approaches, in good agreement with each other, suggest that the dielectric discontinuities enhance counterion condensation for $0<d<\infty$; as $d \rightarrow \infty$, however, the effect of dielectric discontinuities becomes irrelevant at the meanfield level as also expected from Gauss's law (see the relevant discussion in Sec. II).

Using a simple physical picture, we also examine the effect on counterion condensation of charge correlations and backbone-charge distributions. Interestingly, we find that the planar distribution of backbones charges can play an important role: The effect of dielectric discontinuities or image charges depends on how backbone charges are treated (see Sec. III A for details). When the backbone charge is assumed to be smeared out uniformly, then the image charge weakens the attraction of counterions to the surface. When both backbone charges and counterions are treated on equal footing, the image charge can enhance condensation.

Finally, we examine the effect of charge correlations by treating both condensed counterions and backbone charges on equal footing. To this end, we incorporate correlations at the Gaussian level within the two-state model. In the presence of $\mathrm{CaCl}_{2}$ (or $Z: 1$ salts, $Z>1$ ), the (renormalized) charge of a highly charged surface is inverted (when correlations are included), consistent with previous results [9-11]. Interestingly, we find that the correlation effect is more pronounced when the dielectric constant of the surface is lower than in the solution-i.e., $\boldsymbol{\epsilon}_{>}>\boldsymbol{\epsilon}_{<}$-as is often the case. As a result, charge inversion can take place in wider parameter spaces-in this case, the onset of charge inversion takes place at a lower $\mathrm{Ca}^{2+}$ concentration than expected from the case $\epsilon_{>}=\epsilon_{<}$.

This paper is organized as follows: Section II describes charge renormalization at the mean-field level; to this end, a few different versions of mean-field theory are adopted and compared. Section III is devoted to examining the effect of charge correlations on charge renormalization; a particular emphasis is on the interplay between charge correlations and dielectric discontinuities.

\section{MEAN-FIELD THEORY}

\section{A. Poisson-Boltzmann approach and the matching method}

At the mean-field level, the spatial distribution of counterions is described by the PB equation. The PB equation relates the electric potential $\psi(\mathbf{r})$ to the total charge density $\rho(\mathbf{r})$, where $\mathbf{r}$ is the position vector. If $n_{i}(\mathbf{r})$ is the number density of ions of the $i$ th kind and valence $Z_{i}$, it follows

$$
n_{i}(\mathbf{r})=n_{i} \exp \left[-\frac{Z_{i} e \psi(\mathbf{r})}{k_{B} T}\right],
$$

where $e$ is the electronic charge, $k_{B}$ the Boltzmann constant, $T$ the temperature, and finally $n_{i}$ is the bulk concentration of each species. Below we use $n_{i}$ and [ith species] interchangeably, where [ith species] is the bulk concentration of the $i$ th species: $\left[\mathrm{Na}^{+}\right]$is, for example, the bulk concentration of $\mathrm{Na}^{+}$. Obviously the total charge density is given by $\rho(\mathbf{r})$ $=e \sum_{i} Z_{i} n_{i}(\mathbf{r})$. The $\mathrm{PB}$ equation then reads [13]

$$
\boldsymbol{\nabla} \cdot[\epsilon(\mathbf{r}) \boldsymbol{\nabla} \psi]=-4 \pi \rho(\mathbf{r})=-4 \pi e \sum_{i} Z_{i} n_{i}(\mathbf{r}) .
$$

Note that this equation is written in Gaussian units (we adopt Gaussian units throughout this paper). Dielectric discontinuities can be taken into account through spatially varying dielectric constant $\epsilon(\mathbf{r})$.

Without loss of generality, the surface is assumed to be aligned perpendicular to the $x$ axis (cf. Fig. 1). At the meanfield level, the system is essentially one dimensional. In other words, $\psi(\mathbf{r})$ and $\rho(\mathbf{r})$ are functions of $x$ only: $\psi=\psi(x)$ and $\rho=\rho(x)$.

In the matching method, we find a renormalized or an effective charge by matching solutions of the DH equation, with a renormalized charge, and those of $\mathrm{PB}$ equations at large distances from the surface. In other words, the PB approach maps onto the corresponding $\mathrm{DH}$ approach with the bare charge replaced by an effective charge. A simple result for the effective charge density $-e \sigma^{*}$ can be obtained for sufficiently large $\sigma_{0}$ in the limit $d \rightarrow \infty$-i.e., a semi-infinite plate (occupying the space $x<0$ ) in contact with $1: 1$ electrolytes-i.e., $\mathrm{NaCl}$ : It was shown that $\sigma^{*}=\kappa / \pi \ell_{B}$, independent of $\sigma_{0}$ [14]. Here and in what follows, $\ell_{B}$ $=e^{2} / \epsilon_{>} k_{B} T$ is the Bjerrum length, a length scale at which the electrostatic interaction between two charges becomes comparable to the thermal energy $k_{B} T(\approx 7.1 \AA$ at room temperature in water) and $\epsilon_{>}$is the dielectric constant of the solvent (i.e., water); the Debye length $\kappa^{-1}$ is related to ion concentrations through $\kappa^{2}=4 \pi \ell_{B}\left(\left[\mathrm{Na}^{+}\right]+\left[\mathrm{Cl}^{-}\right]\right)$. Finally, the dielectric constant of water at room temperature is known to be 80 . The electrostatic interaction is thus significantly lower in water than in a vacuum.

\section{B. Two-state model}

In a more analytical treatment, we use a two-state model, in which ions are classified as either "free" or "condensed" (i.e., those trapped near the surface). If $Z_{i} e \sigma_{i}$ is the planar charge density of condensed counterions of the $i$ th type, the effective charge density of the surface is then $-e \sigma^{*}=-e\left(\sigma_{0}\right.$ $\left.-\sigma_{1}-Z \sigma_{2}\right)$, where the subscripts 1 and 2 refer to monovalent and $Z$-valent counterions, respectively. Even though we consider only monovalent ions in this section (i.e., $\sigma_{2}=0$ ), we include $Z$-valent counterions for later convenience (cf. Sec. III). The amount of condensed counterions can be obtained by balancing chemical potentials of condensed and free counterions. The chemical potential of free ions is mainly associated with the configurational entropy of mixing: $\mu_{i}^{\text {free }}$ $\simeq k_{B} T \ln \left(n_{i} v_{0}\right)$, where $v_{0}$ is the volume of counterions assumed to be the same for all counterions. The chemical potential of condensed counterions arises from electrostatic interactions and the entropic penalty for condensation. If $\mathcal{F}_{\text {elec }}$ is the electrostatic free energy of the charged surface per area, then the electrostatic chemical potential of condensed counterions of the $i$ th kind is $\mu_{i}^{\text {cond }}=\partial \mathcal{F}_{\text {elec }} / \partial \sigma_{i}$.

The electrostatic free energy of a surface of a planar density $-e \sigma^{*}$ is simply $\mathcal{F}_{\text {elec }}=\frac{1}{2} \int\left(-e \sigma^{*}\right) \psi_{0} d S$, where $\psi_{0}$ is the electrostatic potential evaluated at the surface and $d S$ is a surface element. At the DH level (with a renormalized sur- 
face charge $\sigma^{*}$ ), the electrostatic free energy per unit area is simplified as $\mathcal{F}_{\text {elec }}=\frac{1}{2}\left(-e \sigma^{*}\right) \psi_{0}$, since the charge distribution is assumed to be uniform over the surface. We calculate $\psi_{0}$ by solving $\mathrm{DH}$ equation which is described in details in Appendix A [cf. Eq. (A4)]. We find that, for $\kappa^{-1} \gg \ell_{c}$ [15],

$$
\mathcal{F}_{\text {elec }}=\frac{1}{2}\left(-e \sigma^{*}\right) \psi_{0}=k_{B} T \times 2 \pi \sigma^{* 2} \kappa^{-1} \ell_{B} \frac{\left(\epsilon_{<}+\epsilon_{>} \kappa d\right)}{\left(2 \epsilon_{<}+\epsilon_{>} \kappa d\right)},
$$

where $\epsilon_{<}$and $\epsilon_{>}$are dielectric constants of the bilayer and solvent, respectively, and $\kappa^{-1}$ is the Debye screening length given by $\kappa^{2}=4 \pi \ell_{B}\left[2 n_{1}+Z n_{2}(Z+1)\right]$ with $n_{1}$ and $n_{2}$ the bulk concentration of monovalent and $Z$-valent ions, respectively. We thus have

$$
\frac{\mu_{i}^{\text {cond }}}{k_{B} T}=-4 \pi Z_{i} \sigma^{*} \kappa^{-1} \ell_{B} \frac{\left(\epsilon_{<}+\epsilon_{>} \kappa d\right)}{\left(2 \epsilon_{<}+\epsilon_{>} \kappa d\right)}+\ln \left(\frac{\sigma_{i} v_{0}}{\ell_{c}^{i}}\right),
$$

where $\ell_{i}^{c}$ is the thickness of the condensed layer. The second term in Eq. (4) corresponds to the entropic penalty for confining counterions in a layer of thickness $\ell_{c}^{i}$.

Despite its simplicity, the two-state model suffers a drawback: there can be ambiguity in choosing the thickness of condensed layers $\ell_{c}$ (the superscript $i$ was dropped). In the past, ion sizes were often chosen as $\ell_{c}[1,2]$. While this sounds reasonable, it is not clear whether this choice will lead to $\sigma^{*}$ consistent with the matching method. For a semiinfinite plate $(d \rightarrow \infty)$ in a 1:1 electrolyte, the two approaches can easily be reconciled by mapping the two-state model onto the matching method. In other words, we equate $\sigma^{*}$ $=\kappa / \pi \ell_{B}$ with $\sigma^{*}$ obtained from the two-state model. By noting that $\sigma_{1} \approx \sigma_{0}$ for large $\sigma_{0}$ (this is also the condition under which $\sigma^{*} \approx \kappa / \pi \ell_{B}$, we find

$$
\ell_{c} \approx\left(\frac{\kappa^{-2}}{\lambda}\right)\left(\frac{4}{\exp (4)}\right)
$$

where $1 / \lambda=2 \pi \ell_{B} \sigma_{0}$. Note that this is valid only when $\kappa^{-1}$ $\gg \ell_{c}$; see the relevant discussion in Ref. [15]. This result indicates that $\ell_{c}$ increases quadratically with $\kappa^{-1}$. As a result, $\ell_{c}$ can be much larger than the Gouy-Chapmann length $\lambda$. For finite $d$, dielectric discontinuities will be reflected in $\ell_{c}$. On the other hand, this will not sensitively influence $\sigma^{*}$, since $\sigma^{*}$ varies logarithmically with $\ell_{c}$. In this case, Eq. (5) is expected to be a good approximation for a wide range of parameters (also see Fig. 2). The two-state model and the matching method can thus be used interchangeably. For typical values of parameters $\left(\lambda \sim 10 \AA, \kappa^{-1} \sim 10-100 \AA\right), \ell_{c}$ is smaller than typical ion sizes $a_{0} \sim 5 \AA$. It is thus natural to choose $\ell_{c} \sim a_{0}$ (cf. Figs. 3 and 5).

\section{Dielectric discontinuity}

To test $\ell_{c}$ in Eq. (5) in the presence of dielectric discontinuities, we have calculated $\sigma^{*}$ of the charged surface (at $x=0)$ using the two-state model, with $\ell_{c}$ determined by Eq. (5), and the matching method. We have plotted $\sigma^{*}$ as a function of $\sigma_{0}$ for a few different choices of $\kappa$ (see Fig. 2). We have chosen $d=4 \mathrm{~nm}, \epsilon_{>}=80, \epsilon_{<}=2$, and $T=300 \mathrm{~K}$. In the

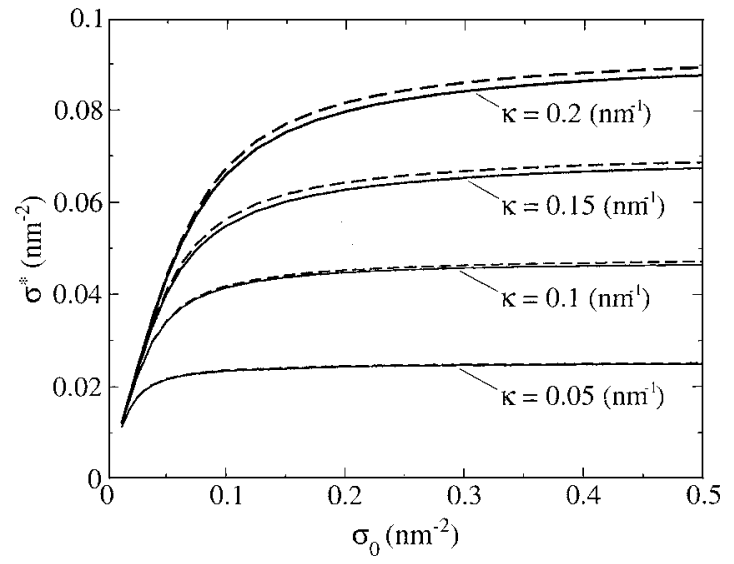

FIG. 2. Effective planar density $\sigma^{*}$ obtained from the two-state model (the dashed line) and the matching method (the solid line). We have chosen $d=4 \mathrm{~nm}, \epsilon_{>}=80, \epsilon_{<}=2$, and $T=300 \mathrm{~K}$; in twostate model calculations, $\ell_{c}$ has been determined by Eq. (5), which was originally obtained for the limit $d \rightarrow \infty$ (or $\kappa d \rightarrow \infty$ ). The two approaches are in good agreement with each other, implying that $\ell_{c}$ in Eq. (5) is valid for a wide range of $\kappa d$ (even when dielectric discontinuities are allowed.)

figure, the two-state model and the matching method are described by the dotted and solid lines, respectively. The agreement between the two approaches is excellent. This justifies our expression for $\ell_{c}$ in Eq. (5) for a wide range of $\epsilon_{<}$(thus $d$ as well), even though it was originally obtained for $d$ $\rightarrow \infty$.

For an asymmetrically charged bilayer of finite thickness $d$, it is useful to examine counterion distributions on both sides of the bilayer. For simplicity we limit ourselves to monovalent cases-i.e., a bilayer immersed in an $\mathrm{NaCl}$ solution. We have used the two-state model to calculate the planar density of condensed counterions $\sigma_{1}$ : the effective planar densities at $x=0$ and $x=-d$ are $\sigma^{*}=\sigma_{0}-\sigma_{1}(x=0)$ and $\sigma^{*}$

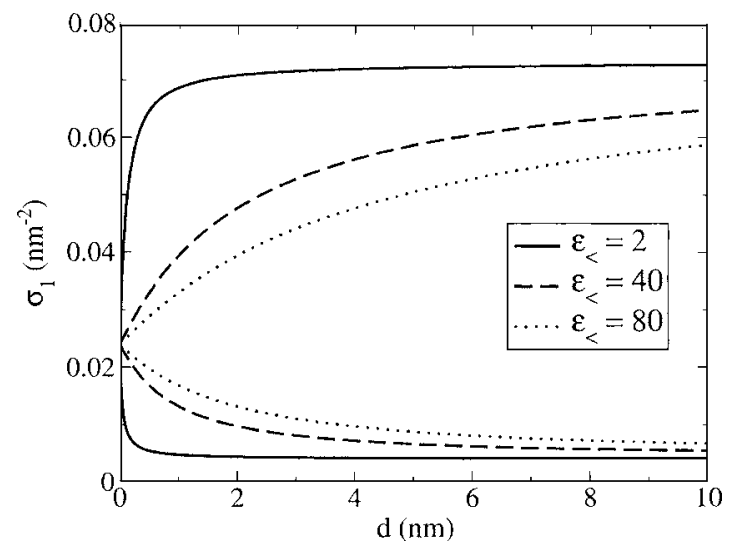

FIG. 3. Planar density of condensed counterions obtained from the two-state model at the charged and neutral surface. We have chosen $\sigma_{0}=0.2 \mathrm{~nm}^{-2}, \epsilon_{>}=80, T=300 \mathrm{~K}, \ell_{c}=5 \AA$, and $\left[\mathrm{Na}^{+}\right]$ $=15 \mathrm{mM}$ (thus $\left[\mathrm{Cl}^{-}\right]=15 \mathrm{mM}$ ). The top (bottom) three curves correspond to the charged (neutral) surface at $x=0(x=-d)$. Note that $\sigma_{1}$ becomes $d$ independent for $\kappa d \epsilon_{>} / \epsilon_{<} \gg 1$ (shown clearly in the figure only for $\epsilon_{<}=2$ ) -in this case, condensation on the neutral surface is minimal and can be ignored (see the text for details). 
$=\sigma_{1}(x=-d)$, respectively. The chemical potentials of counterions at the surfaces at $x=0$ and $x=-d$ are presented in Appendix A. Using these we have calculated $\sigma_{1}(x=0)$ and $\sigma_{1}(x=-d)$ (see Fig. 3) for a few different values of $\epsilon_{<}$; the top three curves are for the charged surface at $x=0$, while the bottom three curves are for the neutral surface at $x=-d$. On the other hand, we have chosen $\sigma_{0}=0.2 \mathrm{~nm}^{-2}, \epsilon_{>}=80, T$ $=300 \mathrm{~K}$, and $\left[\mathrm{Na}^{+}\right]=15 \mathrm{mM}$ (corresponding to $\kappa^{-1}=2.5 \mathrm{~nm}$ ). Finally $\ell_{c} \approx 4 \AA$ in the limit $d \rightarrow \infty$. Since $\epsilon_{>} \kappa d \gg \epsilon_{<}$for the parameters used, $\ell_{c} \approx 4 \AA$ is a good approximation for $d$ $=4 \mathrm{~nm}$. We have thus chosen $\ell_{c}=a_{0}=5 \AA\left(\ell_{c}\right.$ cannot be smaller than the ion size $a_{0} \approx 5 \AA$ ). As shown in the figure, $\sigma_{1}$ tends to get saturated for large $d$ in an $\epsilon_{<}$-dependent way; smaller $d$ is required for smaller $\epsilon_{<}$in a $\kappa$-dependent way. [The $\kappa$ dependence of saturation is not shown in the figure but can be inferred from Eq. (4). What matters is this combination: $\kappa d$.] Also note that $\sigma_{1}(x=-d)$ tends to a finite value, $\sigma_{1}^{\infty}=\lim _{d \rightarrow \infty} \sigma_{1} \approx 0.005 \mathrm{~nm}^{-2}$, as $d$ increases. This is a bit puzzling, since the attraction of counterions to the surface at $x=-d$ is minimal for $\epsilon_{>} \kappa d / \epsilon_{<} \gg 1$, implying that $\sigma_{1}^{\infty} \simeq 0$. As it turns out, $\sigma_{1}^{\infty}(>0)$ reflects $\left[\mathrm{Na}^{+}\right]$-i.e., the bulk $\mathrm{Na}^{+}$ concentration: $\sigma_{1}^{\infty} / \ell_{c}=\left[\mathrm{Na}^{+}\right]$. In other words, the $\mathrm{Na}^{+}$concentration is uniform in the region $x<-d$, meaning that there is no condensation. For typical values of $d(\simeq 4 \mathrm{~nm})$ and $\epsilon_{<}(\simeq 2)$, counterion condensation mainly takes place on the charge surface: $\sigma_{1}(d=4 \mathrm{~nm}) \approx \sigma_{1}^{\infty}$. In what follows, we ignore condensation on the neutral surface.

Our results in Fig. 3 indicate that dielectric discontinuities can enhance counterion condensation for $0<d<\infty$. In the limit $d \rightarrow \infty$, however, the dielectric properties of the plate are not felt by counterions. This is not surprising: Our twostate model in this section suppresses charge fluctuations. In this case, Gauss's law indicates that the electric field cannot penetrate the plate. In other words, the electric field vanishes for $x<0$ independently of $\epsilon_{<}$, as also implied by Eq. (A4) in the limit $d \rightarrow \infty$. This accounts for the $\epsilon_{<}$independence of $\sigma_{1}$ in the limit $d \rightarrow \infty$. Obviously, the effect of the dielectric discontinuity becomes minimal as $d \rightarrow 0$.

To augment our finding of $\epsilon_{<}$-dependent $\sigma_{1}$, we have solved the PB equation [cf. Eqs. (1) and (2)] for a few different choices of $\epsilon_{<}$and plotted our results for $n_{+}(x)$ $\left(=\left[\mathrm{Na}^{+}\right](x)\right.$-i.e., $\mathrm{Na}^{+}$concentration at $\left.x\right)$ in Fig. 4. To this end, we have used essentially the same boundary conditions adopted in Appendix A [see Eqs. (A3a)-(A3c)], except that Eq. (A3c) has been approximated by $\psi(x)=0$ at $x=25 \kappa^{-1}$. We have chosen $d=40 \AA, \epsilon_{>}=80, T=300 \mathrm{~K}$, and $\left[\mathrm{Na}^{+}\right]=1 \mathrm{mM}$. As shown in the figure, $n_{+}(x)$ near the surface is larger for $\epsilon_{<}=2$ than for $\epsilon_{<}=80$. Our results suggest that counterion condensation can be more pronounced for smaller values of $\epsilon_{<}$(as long as $d$ is not too small or too large), in accordance with the results in Fig. 3-note that this happens when $\epsilon_{<}$ $<\epsilon_{>}$, as is the case for a lipid bilayer immersed in water.

Our mean-field results in Figs. 3 and 4 suppress charge correlations and are expected to work well for low electrostatic couplings (e.g., $Z=1$ ). In the next section, we study how charge correlations can influence counterion condensation.

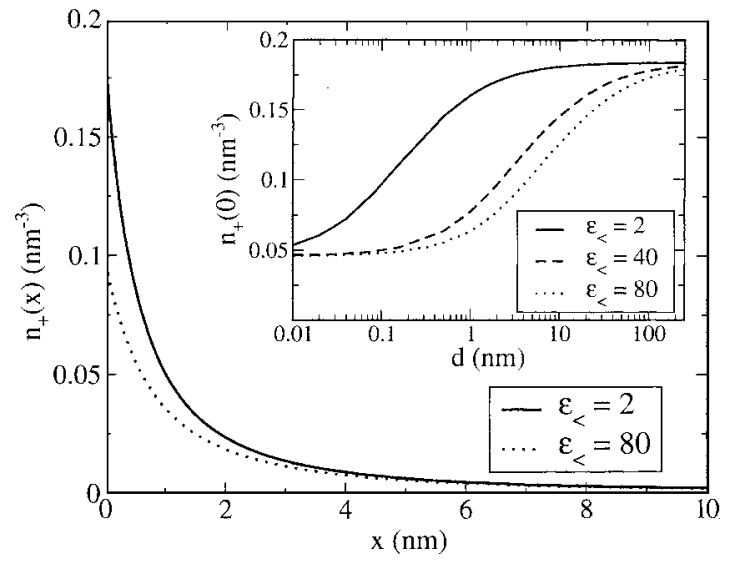

FIG. 4. Spatial distribution of counterions near the charged surface in an ionic solution for different choices of $\epsilon_{<}$, obtained from the Poission-Boltzmann equation. We have chosen $\epsilon_{>}=80, \sigma_{0}$ $=0.2 \mathrm{~nm}^{-2}, d=4 \mathrm{~nm}, T=300 \mathrm{~K}$, and $\left[\mathrm{Na}^{+}\right]=1 \mathrm{mM}$. These results indicate that the dielectric discontinuities at $x=0$ and $x=-d$ enhance counterion condensation at the charged surface $(x=0)$. In the limit $d \rightarrow \infty$ or $d \rightarrow 0$, however, the effect of the dielectric discontinuity becomes minimal (see the inset).

\section{CHARGE CORRELATIONS}

\section{A. Scaling theory: Unscreened cases}

The mean-field approach in the last section indicates that the effect of dielectric discontinuities becomes irrelevant in the limit $d \rightarrow \infty$. This appears to be distinct from those discussed in Refs. $[9,10,16,17]$, which seem to indicate that counterion condensation is diminished by image charges in this limit. It is tempting to attribute the seeming discrepancy to charge correlations which are suppressed in our meanfield calculations. In this subsection, we use simple arguments to discuss the potential effect on counterion condensation of charge correlations and backbone-charge distributions.

Following Refs. [10,18], in the limits $\kappa \rightarrow 0$ and $d \rightarrow \infty$, the electrostatic energy of a single (monovalent) counterion at $\mathbf{r}=(x, y, z)$ due to surface charges at $\mathbf{R}_{\alpha}$ and its image charge is given by

$$
\frac{u(\mathbf{r})}{k_{B} T}=-\ell_{B}(1+\Delta) \sum_{\alpha} \frac{1}{\left|\mathbf{r}-\mathbf{R}_{\alpha}\right|}+\frac{\ell_{B} \Delta}{4 x},
$$

where $\Delta=\left(\epsilon_{>}-\epsilon_{<}\right) /\left(\epsilon_{>}+\epsilon_{<}\right)$. In the continuum limit (i.e., backbone charges are smeared out), $u(\mathbf{r})$ becomes

$$
\frac{u(x)}{k_{B} T}=2 \pi(1+\Delta) \ell_{B} \sigma_{0} x+\frac{\ell_{B} \Delta}{4 x} .
$$

Not surprisingly, $u(x)$ has a minimum at a finite value of $x$ $=x_{\text {min }}$ :

$$
x_{\min }=\sqrt{\frac{\Delta}{8 \pi(1+\Delta) \sigma_{0}}} .
$$

The minimum electrostatic energy is then given by 


$$
\frac{u_{\min }}{k_{B} T}=\ell_{B} \sqrt{\Delta(1+\Delta) \sigma_{0}} .
$$

Clearly, $u_{\text {min }}$ increases as $\Delta$ increases, implying that counterion condensation is diminished by the dielectric jump at the interface $x=0$. This contradicts the PB approach which implies that a single dielectric discontinuity does not affect spatial distributions of counterions for $d \rightarrow \infty$.

The reasoning leading to Eq. (9) is based on the assumption that the backbone charge is smeared out uniformly while the counterion is localized in space. To see the potential effect of backbone-charge distributions more clearly, let us consider only one backbone charge at the origin interacting with a counterion on the $x$ axis. Equation (6) then reduces to

$$
u_{1}(x)=-\ell_{B}(1+\Delta) \frac{1}{x}+\frac{\ell_{B} \Delta}{4 x}=-\ell_{B}\left(1+\frac{3 \Delta}{4}\right) \frac{1}{x} .
$$

Interestingly, this is more attractive for larger $\Delta$ in contrast to what we would expect from Eq. (9) obtained in the continuum limit.

Neither Eq. (7) nor Eq. (10) necessarily represents our system accurately. First, both backbone charges and counterions are mobile (with the former confined to a surface) and can contribute to correlations. They thus have to be treated on equal footing. Furthermore, these equations are based on a one-particle picture, a single counterion interacting with a surface in the former and a counterion interacting with a backbone charge in the latter. "Many-body effects" (e.g., counterion-counterion interactions) can complicate the picture.

Another extreme case that goes beyond the one-particle description amounts to picturing backbone charges and counterions as forming a two-dimensional ordered (crystalline) structure on a square lattice of a lattice constant $a$ at the water-plate interface. An anion is then surrounded by four nearest-neighbor cations and a cation by four nearestneighbor anions. Clearly the energy of the resulting system (per ion) is proportional to $\ell_{B}(1+\Delta)$ :

$$
\begin{aligned}
\frac{u_{\min }}{k_{B} T} & =-\frac{\ell_{B}}{a}(1+\Delta)\left(-4+\frac{4}{\sqrt{2}}+\frac{4}{2}-\frac{8}{\sqrt{5}}+\cdots\right) \\
& \approx-\frac{\ell_{B}}{a}(1+\Delta) \times 1.14 .
\end{aligned}
$$

This is more negative for larger $\Delta$. In this simple picture, counterions are more strongly attracted to the surface when $\epsilon_{<}<\epsilon_{>}$, implying that condensation is enhanced by the dielectric jump at the interface.

This calculation is complimentary to our mean-field approach. If the former is relevant for high electrostatic couplings, the latter is suitable for low couplings. A simpler version of the $\mathrm{PB}$ approach is a capacitor model in which the double layer is approximated by a parallel capacitor: a negatively charged plate at $x=0$ and a positively charged layer at $x=\delta$. In the limit $d \rightarrow \infty$, the image charge of the former (per area) located at $x=0$ is $-e \Delta \sigma_{0}$, which adds to the backbone charge $-e \sigma_{0}$, while the image charge of the latter (per area) located at $x=-\delta$ is $e \Delta \sigma_{0}$. The total electric field felt by the counterion layer at $x=-\delta$ is

$$
E_{x}=\frac{4 \pi e}{\epsilon_{>}}\left[\Delta \sigma_{0}-(1+\Delta) \sigma_{0}\right]=-\frac{4 \pi e}{\epsilon_{>}} \sigma_{0} .
$$

This is independent of $\Delta$ and accounts for our earlier finding that counterion condensation is not influenced by image charges in the limit $d \rightarrow \infty$. Clearly, we need to include correlations to see the effect of image charges on counterion condensation in that limit.

Our simple arguments presented in the last few paragraphs suggest that the effect of dielectric discontinuities on counterion condensation depends on how we treat backbone charges and counterions. This is indeed consistent with a recent paper by Moreira and Netz [18], which shows how surface-charge modulation is intertwined with dielectric discontinuities (also see Naji et al. [19]). The effect of imagecharge repulsions is strongest when the surface charges are assumed to be uniformly smeared out, as also implied by Eq. (7). As a result, the spatial distribution of counterions has a peak at a finite separation from the surface [reminiscent of $x_{\text {min }}$ in our Eq. (7)]. As the surface charge distribution becomes more heterogeneous for a given total surface charge, however, the peak moves towards the surface: $x_{\text {min }}$ is diminished [see their Fig. 4(b) for details] as also implied by our simple scaling analysis. When coupled to correlations, the dielectric jump at the water-bilayer interface can enhance counterion condensation (even in the limit $d \rightarrow \infty$ ).

For a weakly to a moderately highly charged surface, surface charges (both backbone charges and condensed counterions) can be driven by thermal fluctuations, which diminish their lateral ordering. In that case, it is reasonable to consider them as forming a two-dimensional ionic fluid, as compared to a two-dimensional crystal. In the next section, we develop a two-dimensional $\mathrm{DH}$ theory of such an ionic fluid to account for correlations. The resulting approach is distinct from existing approaches $[9,10,16,17]$ in that we treat both backbone charges and counterions on equal footing and consider them as fluctuating objects.

\section{B. Charge correlations and charge inversion}

The previous subsection illustrates the interplay between charge correlations and dielectric discontinuities in determining $u_{\text {min }}$, the minimum electrostatic energy of a counterion near and at an oppositely charged surface. Here, we study how charge correlations can influence counterion condensation. A number of theoretical approaches suggest that charge correlations between condensed counterions (of high valency) can trigger extra condensation, leading to "charge inversion" of a highly charged surface [9-11]. In these approaches [9-11], condensed counterions are considered as forming a strongly correlated liquid on the background of uniformly distributed backbone charges. As evidenced in Sec. III A, backbone-charge distributions can have a nontrivial effect on counterion condensation. In our approach, we treat both backbone charges and condensed counterions on equal footing as fluctuating objects on a plane. To this 
end, we incorporate in-plane charge correlations at the Gaussian level.

In order to set up an effective interaction $\phi\left(\mathbf{r}_{\perp}, \mathbf{r}_{\perp}^{\prime}\right)$ between two charges $e$ 's on the surface at $x=0$ (see Fig. 1), we first need to integrate out degrees of freedom associated with free ions. This can be readily done at the DH level: In the limit $d \rightarrow 0$ (the effect of dielectric discontinuities is irrelevant), this amounts to using a screened electrostatic interac-

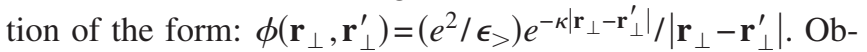
viously, this is the solution of $\left(\nabla^{2}-\kappa^{2}\right) \phi=-\left(4 \pi e^{2} / \epsilon_{>}\right) \delta\left(\mathbf{r}_{\perp}\right.$ $\left.-\mathbf{r}_{\perp}^{\prime}\right)$. However, the presence of dielectric discontinuities (coupled with finite thickness) can easily complicate $\phi\left(\mathbf{r}_{\perp}, \mathbf{r}_{\perp}^{\prime}\right)$. The dielectric discontinuity can be incorporated through a spatially varying dielectric constant $\epsilon(\mathbf{r})$ :

$$
\left[\boldsymbol{\nabla} \cdot \boldsymbol{\epsilon}(\mathbf{r}) \boldsymbol{\nabla}-\epsilon(\mathbf{r}) \kappa^{2}(\mathbf{r})\right] \phi\left(\mathbf{r}, \mathbf{r}^{\prime}\right)=-4 \pi e^{2} \delta\left(\mathbf{r}-\mathbf{r}^{\prime}\right) .
$$

Note that the spatially varying screening length $\kappa^{-1}(\mathbf{r})$ $=\kappa^{-1}(x)$ is to reflect the absence of ions inside the plate (see also Appendix B).

At the DH level, the explicit form of $\phi\left(\mathbf{r}_{\perp}, \mathbf{r}_{\perp}^{\prime}\right)$ can be found without further approximations. By symmetry considerations, we have $\phi\left(\mathbf{r}_{\perp}, \mathbf{r}_{\perp}^{\prime}\right)=\phi\left(\mathbf{r}_{\perp}-\mathbf{r}_{\perp}^{\prime}\right)$-without loss of generality, we can set $\mathbf{r}_{\perp}^{\prime}=0$. In Appendix B, we have solved this equation for our system depicted in Fig. 1 with appropriate boundary conditions (see Appendix B for details). The result is

$$
\phi\left(\mathbf{r}_{\perp}\right)=\int \frac{d^{2} \mathbf{q}}{(2 \pi)^{2}} e^{i \mathbf{q} \cdot \mathbf{r}_{\perp}} \phi(\mathbf{q}),
$$

where $\phi(\mathbf{q})$ is the Fourier transform given by

$$
\beta \phi(\mathbf{q})=\frac{4 \pi \ell_{B}}{\sqrt{\kappa^{2}+q^{2}}+\eta q}\left[1+\xi(\xi-1) \frac{\exp (-2 q d)}{1-\xi^{2} \exp (-2 q d)}\right],
$$

with $\beta=1 / k_{B} T, \eta=\epsilon_{<} / \epsilon_{>}$, and $\xi$ defined as

$$
\xi=\frac{\epsilon_{>} \sqrt{\kappa^{2}+q^{2}}-\epsilon_{<q}}{\epsilon_{>} \sqrt{\kappa^{2}+q^{2}}+\epsilon_{<q}} .
$$

Note that similar issues have been addressed in the literature. For example, $\phi\left(\mathbf{r}_{\perp}\right)$ in the limit $\kappa \rightarrow 0$ was first obtained in Ref. [20]. More recently, Netz considered electrolytes confined to a system of a slab sandwiched between two semiinfinite half spaces, whose dielectric constants can be different from each other [21]. At the Debye-Hückel level, he derived an effective Coulomb interaction between two charges (see $v_{D H}\left(\mathbf{r}, \mathbf{r}^{\prime}\right)$ in Eqs. (A.7)-(A.9) of Ref. [21]). One of the main differences between $\phi$ and $v_{D H}$ is that the latter works for charges that are not on an interface with a dielectric jump, while the former was constructed exclusively for charges on such an interface.

At the Gaussian level, the free energy arising from inplane charge fluctuations on the surface can be readily taken into account. If $\delta \sigma\left(\mathbf{r}_{\perp}\right)$ is the planar charge fluctuation (per $e)$ at $\mathbf{r}_{\perp}=(y, z)$ (normal to the $x$ axis), the Hamiltonian describing fluctuations can be written as [22]

$$
\begin{aligned}
\beta H_{\text {corr }}= & \frac{1}{2} \int d \mathbf{r}_{\perp} \\
& \times d \mathbf{r}_{\perp}^{\prime}\left[\frac{\delta\left(\mathbf{r}_{\perp}-\mathbf{r}_{\perp}^{\prime}\right)}{\chi}+\beta \phi\left(\mathbf{r}_{\perp}-\mathbf{r}_{\perp}^{\prime}\right)\right] \\
& \times \delta \sigma\left(\mathbf{r}_{\perp}\right) \delta \sigma\left(\mathbf{r}_{\perp}^{\prime}\right),
\end{aligned}
$$

where $\chi=\sigma_{0}+\sigma_{1}+Z^{2} \sigma_{2}$ (and $\left.\beta=1 / k_{B} T\right)$. The first term corresponds to the entropic penalty for charge-density fluctuations. While all surface charges are taken into account explicitly through $\sigma\left(\mathbf{r}_{\perp}\right)$, free ions are considered as screening the interaction between surface charges and are taken into account through $\phi$.

In the Fourier space $\mathbf{q}$, conjugate to $\mathbf{r}_{\perp}$, the Hamiltonian (per unit area) is simplified:

$$
\beta \mathcal{H}_{\text {corr }}=\frac{1}{2} \int \frac{d^{2} \mathbf{q}}{(2 \pi)^{2}}\left[\chi^{-1}+\beta \phi(\mathbf{q})\right]|\delta \sigma(\mathbf{q})|^{2} .
$$

By carrying out the Gaussian integrals with respect to $\delta \sigma(\mathbf{q})$, we find the correlation contribution to the free energy $\mathcal{F}_{\text {corr }}$ (after subtracting an appropriate "self-energy" term):

$$
\beta \mathcal{F}_{\text {corr }}=\frac{1}{2} \int \frac{d^{2} \mathbf{q}}{(2 \pi)^{2}}\{\ln [1+\chi \beta \phi(\mathbf{q})]-\chi \beta \phi(\mathbf{q})\} .
$$

In Ref. [23], we derive this result using the Debye-charging process. Note that the correlation free energy in Eq. (19) was constructed so that it vanishes as $\chi \rightarrow 0$ as it should. In practical calculations of $\mathcal{F}_{\text {corr }}$, we cut off high $q$ values by imposing an upper limit for the integral, which will be chosen to be $2 \pi$. On the other hand, the lower limit will be chosen to be 0 .

The charge-correlation contribution to the chemical potential of condensed counterions is simply

$$
\mu_{i}^{\text {corr }}=\frac{\partial \mathcal{F}_{\text {corr }}}{\partial \sigma_{i}} .
$$

The total chemical potential of condensed counterions is then given as the sum of this and the one in Eq. (4).

We have calculated the amount of condensed counterions. In Fig. 5, we have plotted the effective charge density $-e \sigma^{*}$ as a function of $\left[\mathrm{Ca}^{2+}\right]$, the bulk $\mathrm{Ca}^{2+}$ concentration in $\mathrm{mM}$, for various choices of $\epsilon_{<}$. We have chosen $\epsilon_{>}=80, T$ $=300 \mathrm{~K}$, and $\sigma_{0}=0.2 \mathrm{~nm}^{-2}$; we have also assumed that the system contains $100 \mathrm{mM}$ of monovalent ions (or $\left[\mathrm{Na}^{+}\right]$ $=50 \mathrm{mM}$ ). According to Eq. (5), $\ell_{c} \approx 1.2 \AA$ in the absence of $\mathrm{CaCl}_{2}$. While it is possible to generalize Eq. (5) to include $Z: 1$ salts, we rather invoke simplification based on the following physics ground: Unless $\left[\mathrm{Ca}^{2+}\right]$ is too small, $\mathrm{Ca}^{2+}$ can be preferentially condensed onto the surface (see Ref. [24] for details). It is thus reasonable to assume that condensed counterions are mostly $\mathrm{Ca}^{2+}$. In this case, we expect $\ell_{c}$ to be twice the corresponding value for $\mathrm{Na}^{+}$-here we assume that $\kappa$ is mainly determined by $\mathrm{Na}^{+}$. (Recall $\ell_{c} \propto \lambda^{-1}$ and note that $\lambda^{-1}$ for $Z=2$ is twice that for $Z=1$.) The resulting $\ell_{c}$ is smaller than typical ionic sizes $(\sim 5 \AA)$. We have thus chosen $\ell_{c}=5 \AA$. A number of interesting features emerge from the results in the figure. 


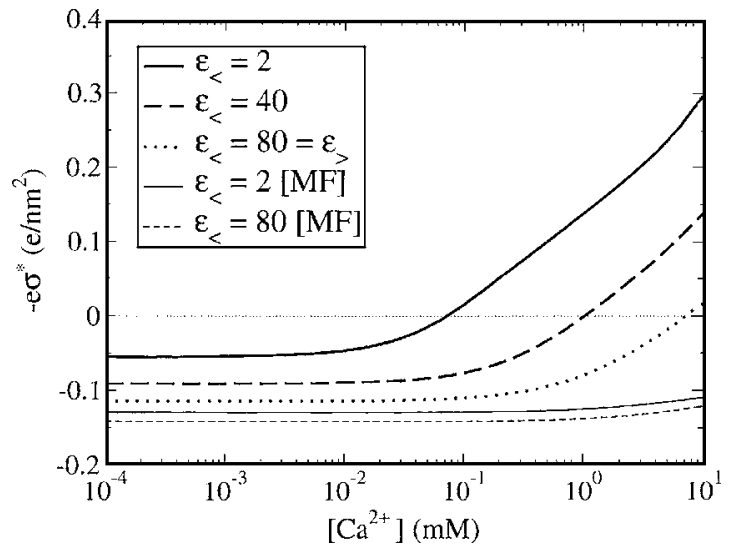

FIG. 5. Effective planar charge density of the charged surface in the presence of $50 \mathrm{mM}$ of $\mathrm{NaCl}$ as a function of $\left[\mathrm{Ca}^{2+}\right]$, the bulk $\mathrm{Ca}^{2+}$ concentration in $\mathrm{mM}$. We have chosen $-e \sigma_{0}=-0.2 e / \mathrm{nm}^{2}, T$ $=300 \mathrm{~K}, \ell_{c}=5 \AA, d=40 \AA$, and $\epsilon_{>}=80$. At low $\mathrm{Ca}^{2+}$ concentrations, the surface is undercharged (i.e., $-e \sigma^{*}<0$ ) but beyond a certain concentration, it is overcharged (i.e., $-e \sigma^{*}>0$ ). The onset concentration for overcharging is sensitive to the dielectric properties of the surface; it is smaller for a smaller value of $\epsilon_{<}$.

First, the results show that charge inversion occurs beyond a certain value of $\left[\mathrm{Ca}^{2+}\right]$ or the onset concentration of $\mathrm{Ca}^{2+}$. This finding is consistent with existing results [9-11]. The effect of charge correlations on condensation is more pronounced when the surface has a lower dielectric constant, as is the case for lipid bilayers in water. As a result, charge inversion occurs for a wider range of $\left[\mathrm{Ca}^{2+}\right]$ for smaller values of $\epsilon_{<}$. Interestingly, the onset concentration $\left(\right.$of $\left.\mathrm{Ca}^{2+}\right)$ is highly sensitive to $\epsilon_{<}$: When the dielectric discontinuity is suppressed $\left(\epsilon_{<}=\epsilon_{>}\right)$[25], the onset of charge inversion takes place at $\left[\mathrm{Ca}^{2+}\right] \approx 7 \mathrm{mM}$. For $\epsilon_{<}=2$, the onset concentration is $\sim 0.1 \mathrm{mM}$, about two orders of magnitude smaller than in the case of $\epsilon_{<}=\epsilon_{>}$.

A related point of interest is that the effect of dielectric discontinuities on condensation is more pronounced for large values of $\left[\mathrm{Ca}^{2+}\right]$. This implies that dielectric discontinuities are more efficiently felt when charge correlations are included. Indeed our mean-field (MF) results, obtained with the two-state model or the matching method introduced in Sec. II, are much less sensitive to $\epsilon_{<}$. Additionally, for large values of $\left[\mathrm{Ca}^{2+}\right]$, the corresponding MF results deviate appreciably from our correlation calculations. This indicates that mean-field approaches can easily break down in the presence of multivalent counterions.

\section{CONCLUSIONS}

To summarize, we have studied how the dielectric properties of a charged surface influence counterion condensation onto the surface. The Poisson-Boltzmann approach and the two-state model indicate that dielectric discontinuities enhance counterion condensation (when the surface has a low dielectric constant, as is the case for a lipid bilayer or other biomolecules in water). This finding appears to contradict earlier results $[9,10,16,17]$ that counterions are pushed away from the surface by image charges. Using simple scaling arguments, we have shown how the effect of image charges is intertwined with backbone-charge distributions. When the backbone charge is assumed to be uniformly smeared out while counterions are localized in space, the image charge tends to diminish counterion condensation $[9,10,16,17]$. When the backbone charge and counterions are treated on equal footing, however, image charges rather enhance counterion condensation. Finally, we have also studied charge inversion of a highly charged surface in a mixture of $\mathrm{NaCl}$ and $\mathrm{CaCl}_{2}$ electrolytes. To this end, we have incorporated inplane charge correlations at the Gaussian level into the twostate model. At a certain value of $\mathrm{Ca}^{2+}$ concentration, the sign of the surface charge is inverted, consistent with earlier results [9-11]. Interestingly, a smaller $\mathrm{Ca}^{2+}$ concentration is required for charge inversion when the surface has a lower dielectric constant; the dielectric discontinuity can lower the onset concentration of $\mathrm{Ca}^{2+}$ dramatically, indicating that the in-plane correlation is more important in the presence of dielectric discontinuities.

\section{ACKNOWLEDGMENTS}

We acknowledge financial supports from the National Sciences and Engineering Council of Canada (NSERC), the Canadian Foundation of Innovation (CFI), and the Ontario Innovation Trust (OIT). We benefited from useful discussions with R. Nets and A. Grosberg.

\section{APPENDIX A}

In this appendix, we recapture some of our results at the Debye-Hückel level, which are relevant for low surface charge densities; we also derive chemical potentials of condensed counterions within the two-state model. Our major conclusion-i.e., enhanced counterion condensation by dielectric discontinuities - can be augmented by the DH calcualtions. To this end, we consider a dielectric plate with thickness $d$ and dielectric constant $\epsilon_{<}$immersed in an ionic solution of dielectric constant $\epsilon_{>}$(typically larger than $\boldsymbol{\epsilon}_{<}$); only one side of the plate at $x=0$ is charged with charge density $-e \sigma$, as illustrated in Fig. 1. The main advantage of examining the DH limit lies in the fact that it allows an analytically tractable analysis of the spatial distribution of counterions. Our DH calculations will thus test more elaborated results reported in the main text. We show that, as $\epsilon_{<}$ $\rightarrow \epsilon_{>}$, the density of counterions reduces near and at the charged surface and increases around the neutral surface. Of course the entire system is always subject to the overall electric neutrality condition

$$
-\int_{-\infty}^{\infty} \rho(x) d x=-e \sigma
$$

where $\rho(x)$ is the total charge density of ions at $x$. This condition implies that at large distances from the surface the electric fields are vanishingly small, since the backbone charge is almost completely screened by surrounding ions.

The linearlized Poisson-Boltzmann equation or the DH equation reads 


$$
\frac{d^{2} \psi}{d x^{2}}=\kappa^{2}(x) \psi(x),
$$

where $\kappa(x)$ is the position-dependent inverse Debye length given by $\quad \kappa(x)=0 \quad$ for $\quad-d<x<0 \quad$ and $\quad \kappa(x) \equiv \kappa$ $=\sqrt{4 \pi \ell_{B}\left[2 n_{1}+Z n_{2}(Z+1)\right]}$ otherwise (refer to Sec. II A). This equation can be solved with appropriate boundary conditions: at water-dielectric interfaces, the electric potential is continuous while the electric field is discontinuous (if $\sigma \neq 0$ or $\epsilon_{>} \neq \epsilon_{<}$. The jump in the normal component of the electric displacement field is $-4 \pi e \sigma$. In addition, we assume that electric potential goes to zero as $x \rightarrow \pm \infty$, which results in zero total charge density at infinity. To summarize, the boundary conditions read

$$
\begin{aligned}
& x=0:\left.\quad \epsilon_{>} \partial_{x} \psi\right|_{0^{+}}-\left.\epsilon_{<} \partial_{x} \psi\right|_{0^{-}}=-4 \pi e \sigma, \\
& x=-d:\left.\quad \epsilon_{<} \partial_{x} \psi\right|_{-d+0^{+}}-\left.\epsilon_{>} \partial_{x} \psi\right|_{-d-0^{+}}=0, \\
& x \rightarrow \pm \infty: \quad \psi=0 .
\end{aligned}
$$

Solving the DH equation subject to these conditions, we find electric potentials in three distinct regions:

$$
\psi(x)= \begin{cases}\frac{-4 \pi e \sigma}{\epsilon_{>} \kappa} \frac{\epsilon_{<}+\epsilon_{>} \kappa d}{2 \epsilon_{<}+\epsilon_{>} \kappa d} e^{-\kappa x}, & x \geqslant 0, \\ \frac{-4 \pi e \sigma}{2 \epsilon_{<}+\epsilon_{>} \kappa d}\left(x+\frac{d}{2}\right)-\frac{2 \pi e \sigma}{\epsilon_{>} \kappa}, & -d<x<0, \\ \frac{-4 \pi e \sigma}{\epsilon_{>} \kappa} \frac{\epsilon_{<}}{2 \epsilon_{<}+\epsilon_{>} \kappa d} e^{\kappa(x+d)}, & x \leqslant-d .\end{cases}
$$

For similar calculations, see Refs. [5,13] and references therein.

From these solutions we can easily infer the effect of dielectric discontinuities on charge distributions. At the $\mathrm{DH}$ level, total charge densities are proportional to electric potentials: $\rho=\left(\epsilon_{>} / 4 \pi\right) \kappa^{2} \psi$. As a result, one can simply write

$$
\begin{gathered}
\rho(x=0) \propto \frac{\epsilon_{<}+\epsilon_{>} \kappa d}{2 \epsilon_{<}+\epsilon_{>} \kappa d}, \\
\rho(x=-d) \propto \frac{\epsilon_{<}}{2 \epsilon_{<}+\epsilon_{>} \kappa d} .
\end{gathered}
$$

It is instructive to take various limits: As $d \rightarrow 0$, the effect of dielectric discontinuities vanishes as expected. For $\epsilon_{>} \kappa d$ $\gg \epsilon_{<},\left(\epsilon_{<}+\epsilon_{>} \kappa d\right) /\left(2 \epsilon_{<}+\epsilon_{>} \kappa d\right) \approx 1$. In this case, dielectric discontinuities become irrelevant. According to Eq. (A5a), the charge density at the right side of the plate (i.e., $x=0$ ) increases as $\epsilon_{<} \rightarrow 0$. In other words, the dielectric jump there enhances the attraction of counterions to the surface. On the other hand, $\rho(x=-d)$ has the opposite behavior: it decreases as $\epsilon_{<}$decreases. This tendency is consistent with the results in Fig. 3.

Now suppose both surfaces are charged with planar densities $\sigma_{L} \equiv \sigma(x=-d)$ and $\sigma_{R} \equiv \sigma(x=0)$. To obtain electric potentials at the two surfaces, note that the DH equation is linear. A linear combination of two DH solutions is a solution of the DH equation. If $\psi_{R}\left(\sigma_{L}=0, \sigma_{R}\right)\left[\psi_{R}\left(\sigma_{L}, \sigma_{R}=0\right)\right]$ is a potential at $x=0$ with $\sigma_{L}=0\left[\sigma_{R}=0\right]$, then the DH potential at the surface is $\psi_{R}\left(\sigma_{L}, \sigma_{R}\right)=\psi_{R}\left(\sigma_{L}=0, \sigma_{R}\right)+\psi_{R}\left(\sigma_{L}, \sigma_{R}=0\right)$. Accordingly, the electric potential at the surface $x=0$ is

$$
\psi_{R}=-\frac{4 \pi e \sigma_{R}}{\epsilon_{>} \kappa} \frac{\left(\epsilon_{<}+\epsilon_{>} \kappa d\right)}{\left(2 \epsilon_{<}+\epsilon_{>} \kappa d\right)}-\frac{4 \pi e \sigma_{L}}{\epsilon_{>} \kappa} \frac{\epsilon_{<}}{\left(2 \epsilon_{<}+\epsilon_{>} \kappa d\right)} .
$$

Note that this satisfies the required boundary conditions at $x=-d$ and $x=0$. As a result, the chemical potential of counterions on the surface is

$$
\begin{aligned}
\frac{\mu_{R}}{k_{B} T}= & -\frac{4 \pi \ell_{B} \sigma_{R}}{\kappa} \frac{\left(\epsilon_{<}+\epsilon_{>} \kappa d\right)}{\left(2 \epsilon_{<}+\epsilon_{>} \kappa d\right)}-\frac{4 \pi \ell_{B} \sigma_{L}}{\kappa} \frac{\epsilon_{<}}{\left(2 \epsilon_{<}+\epsilon_{>} \kappa d\right)} \\
& +\ln \left[\frac{\sigma_{1}(x=0) v_{0}}{\ell_{c}}\right] .
\end{aligned}
$$

Similarly, we find the electric potential at the surface $x=-d$ :

$$
\psi_{L}=-\frac{4 \pi e \sigma_{L}}{\epsilon_{>} \kappa} \frac{\left(\epsilon_{<}+\epsilon_{>} \kappa d\right)}{\left(2 \epsilon_{<}+\epsilon_{>} \kappa d\right)}-\frac{4 \pi e \sigma_{R}}{\epsilon_{>} \kappa} \frac{\epsilon_{<}}{\left(2 \epsilon_{<}+\epsilon_{>} \kappa d\right)} .
$$

This results in

$$
\begin{aligned}
\frac{\mu_{L}}{k_{B} T}= & -\frac{4 \pi \ell_{B} \sigma_{L}}{\kappa} \frac{\left(\epsilon_{<}+\epsilon_{>} \kappa d\right)}{\left(2 \epsilon_{<}+\epsilon_{>} \kappa d\right)}-\frac{4 \pi \ell_{B} \sigma_{R}}{\kappa} \frac{\epsilon_{<}}{\left(2 \epsilon_{<}+\epsilon_{>} \kappa d\right)} \\
& +\ln \left[\frac{\sigma_{1}(x=-d) v_{0}}{\ell_{c}}\right] .
\end{aligned}
$$

The results in Eqs. (A7) and (A9) are used to construct Fig. 3.

\section{APPENDIX B}

In this appendix, we derive the Debye-Hückel Green function $\phi\left(\mathbf{r}_{\perp}-\mathbf{r}_{\perp}^{\prime}\right)$ introduced in Eq. (15) for a dielectric 
plate immersed in an ionic fluid. Note that similar problems have been studied in the literature [20,21]. However, as they are, results presented in these references are not directly applicable to our problem for the reason explained in the text [see the relevant discussion below Eq. (16)]. Here, we present the essential steps leading to $\phi(\mathbf{q})$ in Eq. (15). To this end, we use the DH approach to the system depicted in Fig. 1: a system with a nonuniform dielectric constant $\epsilon(\mathbf{r})$ and a position-dependent screening length $\kappa^{-1}(\mathbf{r})$. To appropriately incorporate dielectric discontinuities within the DH approach, we first consider $\phi\left(\mathbf{r}, \mathbf{r}^{\prime}\right)$ : the electric energy of a point charge $e$ at $\mathbf{r}$ (the field point) due to another point charge $e$ at $\mathbf{r}^{\prime}$ (the source point) or simply the DH Green function. The DH equation for $\phi$ is then

$$
\left[\boldsymbol{\nabla} \cdot \epsilon(\mathbf{r}) \boldsymbol{\nabla}-\epsilon(\mathbf{r}) \kappa^{2}(\mathbf{r})\right] \phi\left(\mathbf{r}, \mathbf{r}^{\prime}\right)=-4 \pi e^{2} \delta\left(\mathbf{r}-\mathbf{r}^{\prime}\right) .
$$

Noting that $\phi$ has a translational invariance in the $y-z$ plane, following Ref. [21], we express $\phi$ as a Fourier transform with respect to $y-y^{\prime}$ and $z-z^{\prime}$ :

$$
\phi\left(\mathbf{r}, \mathbf{r}^{\prime}\right)=\int \frac{d^{2} \mathbf{q}}{(2 \pi)^{2}} e^{i \mathbf{q} \cdot\left(\mathbf{r}_{\perp}-\mathbf{r}_{\perp}^{\prime}\right)} \phi\left(x, x^{\prime}, \mathbf{q}\right),
$$

where $\mathbf{q}$ is the Fourier conjugate to $\mathbf{r}_{\perp}=(y, z)$.

If we use Eq. (B2) in Eq. (B1), we find

$$
\left(\kappa^{2}+q^{2}-\frac{\partial^{2}}{\partial x^{2}}\right) \phi\left(x, x^{\prime}, \mathbf{q}\right)=\frac{4 \pi e^{2}}{\epsilon_{>}} \delta\left(x-x^{\prime}\right), \quad x \geqslant 0,
$$

$$
\begin{gathered}
\left(q^{2}-\frac{\partial^{2}}{\partial x^{2}}\right) \phi\left(x, x^{\prime}, \mathbf{q}\right)=\frac{4 \pi e^{2}}{\epsilon_{<}} \delta\left(x-x^{\prime}\right), \quad-d \leqslant x<0, \\
\left(\kappa^{2}+q^{2}-\frac{\partial^{2}}{\partial x^{2}}\right) \phi\left(x, x^{\prime}, \mathbf{q}\right)=\frac{4 \pi e^{2}}{\epsilon_{>}} \delta\left(x-x^{\prime}\right), \quad x<-d,
\end{gathered}
$$

where $\kappa^{-1}$ is Debye screening length defined in Sec. II A and below Eq. (A2). (Note that similar equations can be found in Ref. [21]. But we use different boundary conditions; see below.) For the computation of $\phi\left(\mathbf{r}_{\perp}, \mathbf{r}_{\perp}^{\prime}\right)$, it suffices to choose $\mathbf{r}^{\prime}=(0,0,0)$ at the water-plate interface. In what follows, we drop $x^{\prime}$ from $\phi\left(x, x^{\prime}, \mathbf{q}\right)$. Up to this point, the field point $\mathbf{r}$ can be anywhere; later it will be chosen to be at the plane $x=0$ [see Eq. (B7)].

The function $\phi(x, \mathbf{q})$ is continuous everywhere but its normal derivatives at $x=0$ and $x=-d$ are discontinuous (as long as $\left.\epsilon_{>} \neq \epsilon_{<}\right)$:

$$
\begin{aligned}
& \lim _{x \rightarrow-0} \boldsymbol{\epsilon}_{<} \frac{\partial}{\partial x} \phi(x, \mathbf{q})-\lim _{x \rightarrow+0} \epsilon_{>} \frac{\partial}{\partial x} \phi(x, \mathbf{q})=4 \pi e^{2}, \\
& \lim _{x \rightarrow-d-0} \epsilon_{>} \frac{\partial}{\partial x} \phi(x, \mathbf{q})-\lim _{x \rightarrow-d+0} \epsilon_{<} \frac{\partial}{\partial x} \phi(x, \mathbf{q})=0 .
\end{aligned}
$$

As in the text, $\epsilon_{<}$and $\epsilon_{>}$are dielectric constants of the plate and water, respectively. The term on the right-hand side of Eq. (B4a) is to reflect the source charge assumed to be located at $x=0$.

With the boundary conditions in Eqs. (B4) and $\phi \rightarrow 0$ at $x= \pm \infty$, we find the solutions of Eqs. (B3) for $\mathbf{q} \neq \mathbf{0}$ [28],

$$
\beta \phi(x, \mathbf{q})= \begin{cases}\frac{2 \pi \ell_{B}}{\sqrt{\kappa^{2}+q^{2}}} e^{-x \sqrt{\kappa^{2}+q^{2}} \frac{(1+\xi)\left(1-\xi e^{-2 q d}\right)}{1-\xi^{2} e^{-2 q d}},} & x \geqslant 0, \\ \frac{2 \pi \ell_{B}}{\eta q}\left(e^{x q}-\xi e^{-x q-2 q d}\right) \frac{1-\xi}{1-\xi^{2} e^{-2 q d}}, & -d \leqslant x<0, \\ \frac{2 \pi \ell_{B}}{\eta q} e^{(x+d)} \sqrt{\kappa^{2}+q^{2}} \frac{\left(1-\xi^{2}\right) e^{-q d}}{1-\xi^{2} e^{-2 q d}}, & x<-d,\end{cases}
$$

where $\beta=1 / k_{B} T, \ell_{B}=e^{2} / \epsilon_{>} k_{B} T, \eta=\epsilon_{<} / \epsilon_{>}$, and $\xi$ is defined in Eq. (16):

$$
\xi=\frac{\epsilon_{>} \sqrt{\kappa^{2}+q^{2}}-\epsilon_{<q}}{\epsilon_{>} \sqrt{\kappa^{2}+q^{2}}+\epsilon_{<q}} .
$$

For the $\mathbf{r}=\left(0, \mathbf{r}_{\perp}\right)$ (both the source and field points are in the same plane: $x=0), \phi(0, \mathbf{q})=\phi(\mathbf{q})$ is simplified as

$$
\beta \phi(\mathbf{q})=\frac{4 \pi \ell_{B}}{\sqrt{\kappa^{2}+q^{2}}+\eta q}\left[1+\xi(\xi-1) \frac{\exp (-2 q d)}{1-\xi^{2} \exp (-2 q d)}\right] .
$$

This is identical to Eq. (15) in Sec III B. 
[1] G. S. Manning, J. Chem. Phys. 51, 954 (1969).

[2] F. Oosawa, Polyelectrolytes (Marcel Dekker, New York, 1971).

[3] See, for example, J.-P. Hansen and H. Löwen, Annu. Rev. Phys. Chem. 51, 209 (2000), and references therein.

[4] For the notion of a "dressed ion" in the context of electrolyte solutions, see R. Kjellander and D. J. Mitchell, J. Chem. Phys. 101, 603 (1994).

[5] R. J. Hunter, Foundations of Colloid Science (Oxford University Press, Oxford, 2001).

[6] P. L. Felgner, Sci. Am. 276(6), 102 (1997).

[7] W. M. Gelbart, R. F. Bruinsma, P. A. Pincus, and V. A. Parsegian, Phys. Today 53(9), 38 (2000).

[8] The outer layer of a red blood cell membrane is coated with sialic acid, which is typically negatively charged. We ignore this extra complexity and assume that the outer layer is neutral.

[9] T. T. Nguyen, A. Yu. Grosberg, and B. I. Shklovskii, Phys. Rev. Lett. 85, 1568 (2000).

[10] T. T. Nguyen, A. Yu. Grosberg, and B. I. Shklovskii, J. Chem. Phys. 113, 1110 (2000)

[11] A. Yu. Grosberg, T. T. Nguyen, and B. I. Shklovskii, Rev. Mod. Phys. 74, 329 (2002).

[12] S. Alexander, P. M. Chaikin, P. Grant, G. L. Morales, P. Pincus, and D. Hone, J. Chem. Phys. 80, 5776 (1984).

[13] See, for example, T. Chou, M. V. Jarić, and E. D. Siggia, Biophys. J. 72, 2042 (1997), and references therein.

[14] L. Bocquet, E. Trizac, and M. Aubouy, J. Chem. Phys. 117, 8138 (2002).

[15] It should be noted that this equation is valid for $\kappa^{-1} \gg \ell_{c}$. Finite thickness of the condensed layer will further complicate $u_{\text {elec }}$. For $d \rightarrow \infty$ and monovalent cases, the electrostatic energy is given by $u_{\text {elec }}=2 \pi \ell_{B} \kappa^{-1} \sigma^{* 2}+2 \pi \ell_{B} \ell_{c}\left(\frac{1}{3} \sigma_{0}^{2}-\sigma_{0} \sigma_{1}+\sigma_{1}^{2}\right)$. It is safe to drop the second term if $\kappa^{-1} \gg \ell_{c}$. For finite $d$, the second term will be complicated by dielectric discontinuities. Nevertheless it is conceivable that this term can easily be dominated by the first one as long as $\kappa^{-1}$ is much larger than $\ell_{c}$.

[16] R. Kjellander and S. Marcelja, Chem. Phys. Lett. 112, 49 (1984).

[17] D. Bratko and B. Jönsson, Chem. Phys. Lett. 128, 449 (1986).

[18] A. G. Moreira and R. R. Netz, Europhys. Lett. 57, 911 (2002).

[19] A. Naji, S. Jungblut, A. G. Moreira, and R. R. Netz (unpublished).

[20] R. Menes, P. Pincus, and B. Stein, Phys. Rev. E 62, 2981 (2000).

[21] R. Netz, Eur. Phys. J. E 5, 189205 (2001).

[22] This is similar, in structure, to Eq. (2.1) in Ref. [20], which describes charge-density fluctuations at the surfaces of a bilayer. In this reference, the effective interaction was obtained for monovalent cases in the limit $\kappa \rightarrow 0$. Ours can thus be considered as a generalization of this. The correlation free energy $\mathcal{F}_{\text {corr }}$ in Eq. (19) obtained with this is identical to the one obtained following the Debye-Charging process. For details, see [23].

[23] It is instructive to derive $\mathcal{F}_{\text {corr }}$ in Eq. (19) using the Debye charging process $[26,27]$. Without loss of generality, we fix an ion of the $i$ th kind and a charge $Z_{i} e$ at the origin $\mathbf{r}_{\perp}^{\prime}=0$ on the surface at $x=0$ and consider the electric potential at $\mathbf{r}_{\perp}$ [denoted by $\left.\Psi_{i}\left(\mathbf{r}_{\perp}, Z_{i}\right)\right]$ due to this ion and other surrounding ions. It should be emphasized that $\Psi_{i}$ is influenced by other surface charges, while $\phi\left(\mathbf{r}_{\perp}\right)$ is not. More precisely, $\Psi_{i}$ can be obtained by integrating out all degrees of freedom associated with surface charges (and free ions) except the one at the origin. At the Debye-Hückel level, we find

$$
\Psi\left(\mathbf{r}_{\perp}, Z_{i}\right)=\frac{Z_{i}}{e} \phi\left(\mathbf{r}_{\perp}\right)-\beta \sum_{j} \sigma_{j} Z_{j}^{2} \int d \mathbf{r}_{\perp}^{\prime} \Psi\left(\mathbf{r}_{\perp}^{\prime}\right) \phi\left(\mathbf{r}_{\perp}^{\prime}-\mathbf{r}_{\perp}\right),
$$

where we dropped a contribution from a finite charge of the surface - the attraction of a counterion to the surface within the two-state model is described by Eq. (3). This leads to

$$
\Psi\left(\mathbf{r}_{\perp}, Z_{i}\right)=\frac{Z_{i}}{e} \int \frac{d \mathbf{q}}{(2 \pi)^{2}} \frac{\phi(\mathbf{q}) e^{i \mathbf{q} \cdot \mathbf{r}_{\perp}}}{1+\beta \sum_{j} \sigma_{j} Z_{j}^{2} \phi(\mathbf{q})} .
$$

Following the Debye charging process [26,27], $\mathcal{F}_{\text {corr }}$ is given by

$$
\mathcal{F}_{\text {corr }}=\sum_{i} Z_{i} e \sigma_{i} \int_{0}^{1}\left[\Psi\left(r_{\perp}, \zeta Z_{i}\right)-\frac{\zeta Z_{i}}{e} \phi\left(r_{\perp}\right)\right]_{r_{\perp} \rightarrow 0} d \zeta .
$$

After the $\zeta$ integral, this becomes the correlation free energy $\mathcal{F}_{\text {corr }}$ in Eq. (19). This is not a coincidence: The Debyecharging calculations and those based on the fluctuation Hamiltonian $\mathcal{H}_{\text {corr }}$ in Eq. (17) are at the same level. The calculations in this endnote justify (at least indirectly) the use of the fluctuation Hamiltonian $\mathcal{H}_{\text {corr }}$ in Eq. (17).

[24] See, for example, J. Israelachvili, Intermolecular and Surface Forces, 2nd ed. (Academic Press, San Diego, 1992).

[25] A similar problem without dielectric discontinuities has been discussed by Y. W. Kim and W. Sung (unpublished).

[26] D. A. Mcquarrie, Statistical Mechanics (Harper \& Row, New York, 1971), Chap. 15.

[27] E. S. Velazquez and L. Blum, Physica A 244, 453 (1997).

[28] Note that the solutions in Eq. (B5) are valid for $\mathbf{q} \neq \mathbf{0}$. In the computation of the correlation free energy in Eq. (19), one can truncate $\mathbf{q} \cong \mathbf{0}$ contributions by imposing a lower limit for the $\mathbf{q}$ integral. On the other hand, $\phi(\mathbf{q})$ obtained in Appendix B remains finite as $\mathbf{q} \rightarrow \mathbf{0}$. Since $d^{2} \mathbf{q}=2 \pi q d q, \mathbf{q} \cong \mathbf{0}$ does not contribute appreciably to the correlation free energy. Thus the lower limit can be chosen to be $\mathbf{q}=\mathbf{0}$ [also see the relevant discussion below Eq. (19)]. 